\title{
Mémoire et imagination dans la Vie de Henry Brulard
}

\section{Zahi ZALLOUA}

la recherche d'une connaissance de soi, ou plutôt en quête d'une
identité personnelle, le narrateur de la Vie de Henry Brulard se
pose implicitement la question suivante: Comment dois-je raconter ma vie? Cette question, à la fois fort pertinente et angoissante, nécessite que le narrateur établisse un rapport clair et distinct vis-à-vis de son passé. Cependant, l'établissement d'un tel rapport est loin d'être évident. Quelle sorte de regard doit-il jeter sur son passé? Quel modèle doit-il suivre? Quels sont les avantages et les inconvénients de certains modes narratifs? Ses réflexions le mènent d'abord au modèle de Chateaubriand, qui lui déplaît immédiatement. A ses yeux, Chateaubriand valorisait à l'excès la subjectivité en la présentant comme centre absolu de son discours: "Je et Moi, ce serait, au talent près, comme $\mathrm{M}$. de Chateaubriand, ce roi des égotistes." Le style pompeux et le moi haïssable de Chateaubriand ${ }^{2}$ deviennent tout ce qu'il faut éviter dans l'activité autobiographique ${ }^{3}$ si le narrateur espère toujours être lu: "Mes Confessions n'existeront donc plus trente ans après avoir été imprimées, si les Je et les Moi assomment trop les lecteurs; et toutefois j'aurai eu le plaisir de les écrire, et de faire à fond mon examen de conscience" (32). Outre l'anti-modèle de Chateaubriand, Stendhal révèle une certaine ambivalence envers Rousseau. Malgré sa grande admiration pour le génie de Rousseau, Stendhal doit 
enfin le dénoncer pour sa glorification excessive du je et du moi: "JeanJacques Rousseau dont bientôt l'emphase m'offensa" (34). ${ }^{4}$

Mais pourquoi, à part les raisons esthétiques, résister à de telles narrations? A l'opposé d'une narration dite romantique à la Chateaubriand/ Rousseau, le narrateur de la Vie de Henry Brulard refuse de falsifier ou de mystifier la représentation de son passé, de transformer son discours autobiographique en un discours littéraire, c'est-à-dire de faire un roman à la première personne. En cela il imite le narrateur de Jacques le fataliste qui s'attache ardemment à la différence entre raconter une histoire vraie et faire un roman: "Il est bien évident que je ne fais pas un roman, puisque je néglige ce qu'un romancier ne manquerait pas d'employer" (Diderot 25). Comme le narrateur diderotien, il insiste à maintes reprises que son travail n'est pas celui d'un faiseur de roman: "Je ferais du roman si je voulais noter ici l'impression que me firent les choses de Paris, impression si fort modifiée depuis" (402); "Je serais obligé de faire du roman et de me chercher à me figurer ce que doit sentir un jeune homme de dix-sept ans, fou du bonheur en s'échappant du couvent, si je voulais parler de mes sensations d'Étroubles au fort de Bard" (421), et encore: "Je mentirais et ferais du roman si j'entreprenais de le détailler" (428). En effet, Stendhal exprime un désir d'authenticité et voudrait bien expulser toute ambiguité de son récit: "Je cherche à détruire le charme, le dazzling des événements, en les considérant ainsi militairement. C'est ma seule ressource pour arriver au vrai dans un sujet sur lequel je ne puis converser avec personne" (40).

Stendhal veut renoncer à ses préjugés, à toutes idées préconçues de soi. Il veut absolument éviter une narration à la Rousseau, qui commence ses Confessions par un jugement préalable vis-à-vis de sa subjectivité. En effet, l'incertitude dans la voix narrative chez Stendhal est évidente dans le passage suivant: "Aurai-je le courage d'écrire ces confessions d'une façon intelligible? Il faut narrer, et j'écris des considérations sur des événements bien petits mais qui précisément à cause de leur taille microscopique ont besoin d'être contés très distinctement" (43). Autrement dit, la définition stendhalienne de soi ne précède pas le projet autobiographique: l'autobiographe se présente comme un sujet en mouvement qui entreprend de se découvrir, de se connaître. ${ }^{5}$ Néanmoins, Stendhal ressent qu'il y a un décalage entre ce que les autres pensent de lui et ce qu'il pense de lui-même, entre l'image que les autres ont de lui et la "véritable" nature de son moi intérieur: "Je passe pour un homme de beaucoup d'es[prit] et fort insensible" (28, je souligne). Se sentant mal compris - une image rousseauiste - et afin d'éviter un autre faux portrait (cette fois-ci de la part du lecteur $\left.{ }^{6}\right)$, le narrateur se met à explorer d'une 
manière authentique les vérités de soi, jusqu'ici cachées à son regard autant qu'à celui d'autrui: 'Qu'ai-je donc été? Je ne le saurais. A quel ami, quelque éclairé qu'il soit, puis-je le demander? M. di Fior[i] lui-même ne pourrait me donner d'avis. A quel ami ai-je jamais dit un mot de mes chagrins d'amour?" (29).

A la recherche d'une narration authentique, il contemple même la possibilité de raconter à la troisième personne: "On pourrait écrire, il est vrai, en se servant de la troisième personne, il fit, il dit" (30). Mais cette idée est rapidement rejetée puisqu'elle ne permettrait pas d'explorer ses états d'âme, l'intériorité de son moi: "Oui, mais comment rendre compte des mouvements intérieurs de l'âme?" (30, je souligne). Mais bien que le narrateur n'adopte pas cette narration à la troisième personne, la narration de son moi intime se pose comme étant plus proche de la philosophie que de la littérature:

A force d'employer des méthodes philosophiques, par exemple à force de classer mes amis de jeunesse par genres, comme $\mathrm{M}$. Adrien de Jussieu fait pour ses plantes (en botanique), je cherche à atteindre cette vérité qui me fuit. Je m'aperçois que ce que je prenais pour de hautes montagnes, en 1800, n'étaient la plupart que des taupinières; mais c'est une découverte que je n'ai faite que bien tard. (43-44)

Il est clair que l'autobiographie de Stendhal prétend être du côté du vrai et non pas de la fiction. En traitant son passé comme objet d'observationc'est-à-dire comme quelque chose à élucider, à déchiffrer et à exprimer avec netteté-Stendhal perpétue sa distinction initiale entre sujet et objet: "Je vais avoir cinquante ans, il serait bien temps de me connaître. Qu'ai-je été, que suis-je, en vérité je serais bien embarrassé de le dire" (28). Les questions "qu'ai-je été?" et "que suis-je?" donnent l'apparence d'une distinction nette et statique entre la conscience et la facticité du narrateur, entre le je raconteur (présent) et le je raconté (passé). Toutefois, nous allons voir que la relation entre conscience et facticité est beaucoup plus complexe et dynamique que celle envisagée initialement par le narrateur.

Dans la Vie de Henry Brulard, le narrateur, en tant qu'une conscience réfléchissante, semble donc avoir évacué toute imagination de son discours. Ce qui l'intéresse est de clarifier sa mémoire d'une manière rigoureuse et non de mystifier ses souvenirs par un langage romanesque. Cette critique préliminaire de l'imagination reflète la prise de conscience adulte de l'existence d'un décalage entre imagination et réalité: "Je trouvai 
la réalité bien au-dessous des folles images de mon imagination. [...] Ce désappointement, je l'ai eu à peu près dans tout le courant de ma vie" (226). Le narrateur revient plusieurs fois sur le fait qu'il y a un manque de correspondance entre le royaume de l'imaginaire et celui du réel. Possédant une imagination débridée, le jeune Beyle se croit un personnage littéraire, pour lui le réel et l'imaginaire ne se distinguent plus:

Pour un rien, par exemple une porte à demi ouverte la nuit, je me figurais deux hommes armés, m'attendant pour m'empêcher d'arriver à une fenêtre donnant sur une galerie où je voyais ma maîtresse. C'était une illusion qu'un homme sage comme Abraham Constantin, mon ami, n'aurait point eue. Mais au bout de peu de secondes (quatre ou cinq tout au plus) le sacrifice de ma vie était fait et parfait, et je me précipitais comme un héros au-devant des deux ennemis qui se changeaient en une porte à demi-fermée. (44)

A première vue, le narrateur semble se moquer de la pensée romanesque de sa jeunesse, mais cette distance ironique entre le narrateur et le jeune Stendhal est rapidement réduite lorsque le narrateur nous confesse que ce désir de mystifier les choses ne lui est pas non plus tout à fait étranger. Le romanesque est toujours prêt à envahir la réalité du narrateur: "Il n'y a pas deux mois qu'une chose de ce genre [...] m'est encore arrivée. Le sacrifice était fait et tout le courage nécessaire était présent, quand après vingt heures je me suis aperçu, en relisant une lettre mal lue [... que c'était une illusion" (44).

Malgré son esprit rêveur, le narrateur n'a guère envie de répéter les erreurs de sa jeunesse. Il y a donc une extrême prudence dans la démarche stendhalienne. Ayant critiqué le pouvoir trompeur de l'imagination, il envisage une quête de soi basée sur une exploration de sa mémoire, c'est-àdire sur des découvertes et non des fabrications. Dans cette entreprise, la fonction de l'écriture semble être à première vue instrumentale. Autrement dit, l'écriture sert au narrateur essentiellement à structurer sa vie, à exprimer le contenu de la mémoire, et à classifier les vécus du narrateur: "Donc, en classant ma vie comme une collection de plantes, je trouverai [...]" (44).

Ainsi, bien que le narrateur de la Vie de Henry Brulard se soit volontairement décentré (dans le refus de s'engager dans un discours romantique), sa voix reste apparemment stable; la narration choisie, qui émerge après deux chapitres de spéculation, n'est ni trop centrée sur le moi (une vision romantique) ni trop détachée de celui-ci (une vision 
cartésienne). En conséquence, le narrateur, nous semble-t-il, commence son autobiographie au troisième chapitre, après avoir annoncé à la fin du deuxième chapitre: "Après tant de considérations générales je vais naître" (45, je souligne).

En écrivant son autobiographie, le narrateur se rend compte du pouvoir de l'écriture, qui permet de faire resplendir des souvenirs et d'élucider les événements du passé: “En écrivant ma vie en 1835, j’y fais bien des découvertes"; "En écrivant ceci l'image de l'arbre de la Fraternité apparaît à mes yeux, ma mémoire fait des découvertes" (326), et encore: “Tout ceci ce sont des découvertes que je fais en écrivant" (435). Ainsi, bien que l'écriture ait une fonction instrumentale (elle l'aide dans la représentation de ses souvenirs), elle joue souvent un rôle fort plus actif dans leur déclenchement. Cet aspect important de l'écriture rend donc problématique une compréhension statique et simplificatrice de la relation entre écriture et mémoire.

L'écriture révèle au narrateur certaines vérités de son passé: "Je ne vois la vérité nettement sur la plupart de ces choses qu'en les écrivant en 1835, tant elles ont été enveloppées jusqu'ici de l'auréole de la jeunesse, provenant de l'extrême vivacité des sensations" (43). Submergé par la prolifération des souvenirs déclenchés par l'écriture, la force de leur résurrection risque d'engendrer une déstabilisation du projet autobiographique: "les details [...] m'arrivent en foule" (82); "Les souvenirs se multiplient sous ma plume" (283). Effectivement, la prolifération de digressions, ce que Béatrice Didier nomme "une écriture de rupture" (252), menace de transgresser les limites établies par le narrateur. ${ }^{7}$ Le modernisme de l'écriture stendhalienne a été bien observé par Richard N. Coe. Dans "Stendhal, Rousseau, and the Search for the Self," Coe constate que Stendhal prévoit "l'écriture automatique" des surréalistes: "It is almost as though, the better part of a century before Breton and Soupault, he had discovered the principle of automatic writing-speed, in this case, furnishing the element of automatism" (29). Dépassant sa fonction utilitaire, l'écriture stendhalienne devient une activité créatrice et dans ce sens annonce également la "mémoire involontaire" proustienne, où la mémoire n'est plus conçue comme un "bloc monolithique" (Del Litto ix) transparent à la conscience du narrateur mais comme un domaine dont la vraie profondeur et la richesse ne se révèlent que momentanément à travers les digressions hasardeuses de l'écriture. Il y a donc une certaine ambivalence envers l'écriture. Elle l'aide à ressusciter ses souvenirs, mais en même temps elle risque, en frustrant constamment son projet de voir clair, d'engendrer un double décentrement (le premier décentrement fut effectué 
de manière délibérée par le narrateur, avec son refus de perpétuer le culte du moi). La peur d'un déplacement textuel est signalée par les réactions du narrateur à la menace des digressions, par la multiplication des "je m'égare," "je me laisse emporter," etc.

Le narrateur est également conscient du danger de laisser l'écriture s'engager dans le domaine du romanesque, c'est-à-dire celui de l'imagination. Comme le remarque Béatrice Didier, "L'écriture doit suivre les interruptions de la mémoire, ne pas chercher à remplir les vides" (253). Stendhal s'oppose à une solution rousseauiste aux lacunes de la mémoire. Il ne veut pas céder à la tentation du romanesque, à la "rousseauisation" de la réalités et ce qui résulterait en une transformation de l'autobiographie en un travail romanesque. Bien que sa mémoire soit impuissante à donner une vision unifiée de la subjectivité (l'image de soi qui surgit de sa mémoire n'est qu'un amas de souvenirs sans cohérence profonde), le narrateur ne cède pas à la tentation de combler les vides par l'imagination.

Autour de cette réflexion sur la nature lacunaire de la mémoire, une métaphore significative se produit: "A côté des images les plus claires je trouve des manques dans ce souvenir, c'est comme une fresque dont de grands morceaux seraient tombés" (130). Stendhal reprend cette métaphore un peu plus loin dans le récit: "Ce sont de grands morceaux de fresques sur un mur, qui depuis longtemps oubliés apparaissent tout à coup, et à côté de ces morceaux bien conservés sont [...] de grands espaces où l'on ne voit que la brique du mur" (142). En faisant "allusion aux fresques du Campo Santo de Pise et à leur état actuel" (338), le narrateur compare l'état de sa mémoire à la condition fragmentée d'une fresque afin de souligner qu'en dépit d'une image incompréhensible de soi il ne s'agirait pas ici de faire une restauration traditionnelle de la fresque, tentation à laquelle un Rousseau n'aurait pas résisté. Comme nous l'avons déjà souligné, le narrateur de la Vie de Henry Brulard est tout à fait catégorique dans son refus de faire un roman: "La fresque est tombée en cet endroit et je ne serais qu'un plat romancier [... s si j'entreprenais d'y suppléer" (338).

Mais, bien qu'il affirme que son texte n'est que le produit de sa mémoire - "ce livre-ci [est] fait uniquement avec ma mémoire" (394)nous voudrions suggérer que Stendhal n'a pas évacué entièrement l'imagination de son discours autobiographique, et qu'elle joue au contraire un rôle fort considérable. A la différence de Rousseau, dont la figure n'arrête pas de hanter la Vie de Henry Brulard', l'imagination chez Stendhal est, à notre avis, beaucoup plus subtile. Rappelons brièvement la critique préliminaire de l'imagination. Selon le narrateur, le pouvoir de l'imagination mène souvent à une mystification de soi et à une déformation de la réalité. 
Nous proposons ici de reconsidérer le rôle de l'imagination à la lumière de l'attitude paradoxale qu'affiche le narrateur par rapport à son identité personnelle. Y-a-t-il une continuité et/ou une discontinuité du "je" stendhalien?

Commençons par une analyse de ces énonces apparemment contradictoires:

1. En l'aimant à six ans peut-être, 1789, j'avais absolument le même caractère qu'en 1828 en aimant à la fureur Albertine de Rubempré. Ma manière d'aller à la chasse au bonheur n'avait au fond nullement changé. (51)

2. Je conclus de ce souvenir, si présent à mes yeux, qu'en 1793, il y a quarante-deux ans, j'allais à la chasse du bonheur précisément comme aujourd'hui, en d'autres termes plus communs mon caractère était absolument le même qu'aujourd'hui. (123)

3. Je fus saisi d'un des plus vifs mouvements de joie que j'aie éprouvés en ma vie. Le lecteur pensera peut-être que je suis cruel mais tel j'étais à dix ans tel je suis à cinquante-deux. (121)

4. Pour peu que le lecteur ait l'âme commune, il s'imaginera que cette longue digression a pour but de cacher ma honte d'avoir écrit cella. Il se trompe: je suis un autre homme. (391)

5. Depuis trente ans au moins j'ai oublié cette époque si ridicule de mon premier voyage à Paris, sachant en gros qu'il n'y avait qu'à siffler je n'y arrêtais pas ma pensée. Il n'y a pas huit jours que j'y pense de nouveau; et s'il y a une prévention dans ce que j'écris elle est contre le Brulard de ce temps-là. (408-9)

Les énoncés 1,2 et 3 affirment une permanence d'identité de la part du narrateur, tandis que les énoncés 4 et 5 suggèrent un manque de cohérence entre le je raconté (le jeune Stendhal) et le je raconteur (le narrateur). De nombreux critiques ont minimisé les apparentes discontinuités, choisissant d'accentuer la continuité d'identité. Emile J. Talbot, par exemple, écrit: "There are aspects of the human personality, he [Stendhal] understands are discontinuous. The more important character traits, however, are remarkable for their persistence" (99). Cette interprétation nous paraît cependant problématique. Pourquoi ces traits de caractère sont-ils persistants? En refusant de mettre en cause la nature ou l'origine de ces 
traits, Talbot semble suggérer l'existence d'une "vraie" nature stendhalienne, c'est-à-dire l'existence d'une espèce d'essence qui persiste au long du récit autobiographique. Mais, à notre avis, ce problème de continuité et discontinuité d'identité pourrait être élucidé sans faire appel à une explication essentialiste si le problème était reconsidéré à la lumière d'une relecture de la fonction de l'imagination chez Stendhal.

Dans les énoncés 1 et 2 , nous avons une identification nette entre je raconteur et je raconté; les deux je ont le "même caractère." En partageant le même projet de "la chasse au bonheur," Stendhal souligne sa joie de vivre, son enthousiasme pour la vie. ${ }^{10}$ Cette insistance sur la "chasse au bonheur" révèle au lecteur la primauté qu'attribue Stendhal au plaisir. Et c'est en effet ce choix de projet qui l'a défini auparavant-et qui continue à le définir au présent—comme hédoniste, au sens bien sûr philosophique du terme. Dans l'énoncé 1 , le narrateur arrive à trouver une correspondance entre l'amour qu'il a éprouvé envers sa mère et Albertine de Rubempré. Pourtant, il n'est pas question ici de découvrir un trait essentiel de caractère partagé par ces deux femmes. En établissant une distinction entre le sujet du désir (Stendhal-y compris le je raconteur et le je raconté) et l'objet du désir (les femmes de sa vie), nous voudrions mettre en lumière le fait que c'est le sujet du désir qui en fait rend cet énoncé particulièrement significatif. Cette relation imaginaire entre les deux femmes a comme fonction de recentrer Stendhal. En investissant son passé d'une imagination créatrice, le narrateur établit un espace dans lequel une cohérence de soi pourrait s'établir. Certes, cette identité n'est pas "ready made," mais sa construction ne correspond pas non plus à une mystification de la réalité: elle n'est pas une création ex nihilo de la part du narrateur. En d'autres termes, l'identité personnelle n'est pas donnée, mais est le résultat d'un travail d'imagination. Ainsi "découvrir" et "créer" ne sont pas en opposition dans l'univers stendhalien, mais réconciliés grâce à l'imagination créatrice du narrateur.

Dans l'énoncé 3 , nous avons une illustration évidente de la force de l'énergie stendhalienne: "Je fus saisi d'un des plus vifs mouvements de joie que j'aie éprouvés en ma vie.” Après avoir souhaité la mort de Louis XVI: "J'espère que le traître aura été exécuté" (121), la réalisation de son souhait produit en lui un "mouvement de joie" qui pourrait, remarque le narrateur, paraître à un lecteur indiligent comme un sentiment excessif: "Le lecteur pensera peut-être que je suis cruel" (121). Cependant, Stendhal maintient que c'est cette volonté pleine de passion qui l'a défini-et qui continue à le définir-comme un homme révolté, c'est-à-dire un homme qui s'oppose à la tyrannie de l'indifférence et à toute pensée réactionnaire. 
C'est l'intensité, et non pas la modération, qui l'aide à se différencier des autres, à affirmer sa propre volonté de puissance. De la haine du père à l'amour de la mère:

Je voulais couvrir ma mère de baisers et qu'il n'y eût pas de vêtements. Elle m'aimait à la passion et m'embrassait souvent, je lui rendais ses baisers avec un tel feu qu'elle était souvent obligée de s'en aller. J'abhorrais mon père quand il venait interrompre nos baisers. Je voulais toujours les lui donner à la gorge. $(51)^{11}$

Stendhal tire un certain plaisir de l'intensité violente de ses émotions. Ce côté émotionnel de sa personnalité-qui exprime sa singularité-le narrateur ne veut guère le changer. Pour cette raison, il nous semble raisonnable de voir un rapprochement entre ses deux je: une continuité entre le narrateur mûri et le jeune Stendhal.

Dans les énoncés 4 et 5 , le narrateur, par contre, marque sa différence entre sa jeunesse et sa condition présente, et démontre que sa conscience n'est pas identique à son passé, qu'elle dépasse sa facticité: “je suis un autre homme" et "s'il y a une prévention dans ce que j'écris elle est contre le Brulard de ce temps-là." L'indétermination de sa conscience semble lui permettre à la fois de s'identifier et de se distancier de son je raconté, c'est-à-dire de se rapprocher et de s'éloigner de son soi antérieur. Et c'est précisément ce manque de coïncidence ontologique entre je raconteur (conscience) et le je raconté (facticité) qui rend possible, nous semble-t-il, l'activité d'interprétation: “Je rumine sans cesse sur ce qui m'intéresse, à force de le regarder dans des positions d' âme différentes je finis par y voir du nouveau, et je le fais changer d'aspect" (292). En multipliant ses perspectives, son imagination cherche constamment à repenser le passé. Ne possédant pas de valeur intrinsèque, le passé devient pour le narrateur l'objet inépuisable d'interprétations (et, comme nous avons déjà vu, l'interprétation du passé chez Stendhal est une activité profondément créatrice-un processus d'autocréation.).

Mais si la conscience humaine dépasse toujours ce que l'individu est, comment serait-il possible pour le narrateur d'achever son autobiographie? En terminant l'autobiographie, le sujet référentiel (c'est-à-dire le sujet existentiel ou extratextuel) du discours autobiographique ne déclarerait-il pas sa propre mort? Outre ces préoccupations formelles concernant le dénouement autobiographique, la problématique stendhalienne se situe autour de la question de l'amour"12: ce "bonheur fou" (434). Après avoir révélé son amour pour Angela Pietragrua, le narrateur nous prévoit qu'il 
aura grande difficulté à faire une narration raisonnable de ses chers souvenirs à Milan. Son bonheur, nous dit-il, "surpasse le disant" (434), et il termine son autobiographie en notant: "On gâte des sentiments si tendres à les raconter en détail" (435).

Mais que faut-il entendre par ce dénouement fort ambigu? Est-ce une réflexion critique sur la nature du langage, une mise en cause de la relation entre les mots et les choses? La fin met-elle en relief qu'il y a des sentiments qui sont impossibles à exprimer par des mots, un manque de correspondance parfaite entre le langage et la réalité? Ces questions nous renvoient au début du récit. Si le langage est essentiellement imparfait dans la représentation des sentiments, comment devons-nous traiter tout ce que le narrateur vient jusqu'ici de nous raconter? Face au problème de l'indicible (ce qui est proprement inénarrable), comment le lecteur peut-il éviter une vision sceptique de l'autobiographie? Ces questions, bien qu'elles soient à un certain niveau nécessaires et justifiables, nous semblent fondées sur une lecture limitée du dénouement comme une mise en cause du langage.

Béatrice Didier nous offre une autre lecture. Elle reprend et situe la question du dénouement dans le contexte d'une critique du romanesque. Le refus stendhalien de décrire le bonheur illustre une fois de plus son opposition à l'anti-modèle Rousseau, qui dans les Confessions ne démontra aucun souci en racontant son bonheur aux Charmettes. Cette résistance stendhalienne au "pathos romantique" de raconter ses sentiments les plus profonds continue à contribuer à la subversion du romanesque. Au lieu de faire un discours autour de la représentation de son amour, Stendhal arrive à souligner l'intensité de son bonheur, paradoxalement, par l'absence d'une représentation de son amour.

Ajoutant à la lecture de Didier, nous pouvons voir que la "peur du Romanesque" devient pour Stendhal une peur de la pétrification. Stendhal ne veut pas que ces sentiments soient appropriés par le langage; cette possibilité lui fait absolument horreur. S'il les écrit, ses sentiments (ses vécus les plus chers) ne lui appartiendront plus et par la suite il risquera de devenir aliéné de son propre bonheur. Ainsi que l'a très bien fait ressortir Michel Crouzet, c'est dans la communication que "le plus précieux" perdrait sa singularité et "deviendrait commun" (125). Selon cette perspective, le dénouement nous semble fort ironique, puisqu'il finit par mettre en cause le projet même de l'autobiographie: une poétique de la confession. Au lieu de nous présenter des nouvelles Confessions, Stendhal semble produire des anti-Confessions. Mais ici l'ironie pourrait également être considérée comme une stratégie défensive de la part de Stendhal, comme une façon 
de protéger son moi intérieur du regard du lecteur. L'énoncé suivant, qui met en lumière la fragilité de son moi intérieur, semble préfigurer ce développement: "Pendant tout le cours de ma vie, je n'ai jamais parlé de la chose pour laquelle j'étais passionné, la moindre objection m'eût percé le cœur" (195). De plus, le pseudonyme de Henry Brulard n'annonce-t-il pas dès la lecture du titre de l'autobiographie une poétique de la dissimulation?

Alors, l'ironie fonctionne-t-elle comme une subversion du romanesque (une force déstabilisatrice qui met en cause les conventions de l'autobiographie et les normes de la lecture) ou comme un contre-discours ayant comme but la protection du moi intérieur (une force qui risque paradoxalement de problématiser la construction de soi)? Toujours fort conscient de l'attitude de son lecteur, Stendhal écrit: "O lecteur froid, excusez ma mémoire, ou plutôt sautez cinquante pages" (435). Ce lecteur impatient est sans doute déconcerté par la résistance stendhalienne à la clôture. Mais en critiquant un tel lecteur, Stendhal ne fait-il pas implicitement appel aux lecteurs à venir, à ses "happy few," pour qui une lecture ne s'agirait pas de maîtriser le récit en donnant un sens définitif à la Vie de Henry Brulard?

Pour notre part, nous avons tenté de repenser le projet autobiographique de Stendhal en démontrant que les questions préliminaires du narrateur: "qu'ai-je été?" et "que suis-je?" furent essentiellement mal posées. Malgré son refus du romantisme, son refus de s'engager dans un culte du moi, Stendhal est loin d'être le narrateur cartésien comme il voudrait nous le faire croire au début du récit. Dans son projet de voir clair, basé seulement sur une exploration de la mémoire, Stendhal n'a pas réussi à localiser une image cohérente de soi. Au contraire, l'originalité de la Vie de Henry Brulard réside dans le fait d'avoir mis en relief la fonction créatrice de l'imagination dans la production/découverte du "je" stendhalien. Cependant, cette image de Stendhal, étant devenue inséparable du processus autobiographique, demeure, nous semble-t-il, comme la Vie de Henry Brulard, pour toujours inachevée. ${ }^{13}$

Princeton University 


\section{Notes}

1. Stendhal, Vie de Henry Brulard (Paris: Gallimard, 1973) 30. Toutes mes références sont à cette édition et j'indiquerai dans le texte les pages auxquelles elles renvoient.

2. Le narrateur de la Vie de Henry Brulard n'éprouve que mépris pour son prédécesseur: "En paraissant vers 1803 le Génie du Christianisme m'a semblé ridicule" (31). Ce mépris rappelle celui qu'il ressent pour son père Chérubin Beyle. Il y a donc un double renoncement à la figure paternelle: celle du père biologique et celle du père du romantisme. En refusant le père (biologique et symbolique), Stendhal souligne son manque de détermination et dans ce sens renvoie au projet essentiel de l'autobiographe, celui de donner un sens à sa vie, de s'autocréer.

3. Dans "Stendahl et les problèmes de l'autobiograhpie," Philippe Lejeune définit le discours autobiographique de la manière suivante: "J'entends ici naturellement 'autobiographique' au sens étroit et précis que j'ai défini: histoire synthétique d'un individu écrite par lui-même, histoire qui implique retour à l'origine, c'est-à-dire au récit d'enfance"'(25).

4. Plus tard dans le récit, le narrateur ajoute à sa critique de Rousseau: "Il faudra peut-être relire et corriger ce passage, contre mon dessein, de peur de mentir avec artifice comme J. J. Rousseau" (412). Il est important de noter, cependant, que Stendhal prend ses distances avec Rousseau, sans pourtant le renier jamais complètement.

5. En posant la question: "Quel œil peut se voir soi-même?, "Stendhal met en cause l'existence d'un "je" transcendantal transparent à sa conscience (31). Dans l'énoncé suivant, Jean-Paul Sartre exprimera, à notre avis, ce qui est déjà implicite chez Stendhal: "Il n'y a pas de Je sur le plan irréfléchi " (32). C'est-à-dire que le "je" du narrateur/autobiographe a un statut dérivatif et non primordial. Pour une lecture phénoménologique de la Vie de Henry Brulard, on pourra consulter avec profit Time and Narrative in Stendahl.

6. Le narrateur est fort conscient du regard du lecteur; il se sent constamment jugé par lui, essayant d'anticiper la réaction du lecteur, le narrateur remarque: "Je pourrais remplir dix pages des détails de cette soirée, mais si les lecteurs de 1880 sont aussi étiolés que la bonne compagnie de 1835 , la scène comme le héros leur inspireront un sentiment d'éloignement profond" (122).

7. Dès le premier chapitre, le narrateur semble anticiper ce danger: "Mais je me laisse emporter, je m'égare, je serai inintelligible si je ne suis 
pas l'ordre des temps, et d'ailleurs les circonstances ne me reviendront pas si bien" (34).

8. "J'écrivais mes Confessions déjà vieux, et dégoûté des vains plaisirs de la vie que j'avais tous effleurés et dont mon coeur avait bien senti le vide. Je les écrivais de mémoire; cette mémoire me manquait souvent ou ne me fournissait que des souvenirs imparfaits et $\mathrm{j}$ 'en remplissais les lacunes par des détails que j'imaginais en supplément de ces souvenirs " (Jean-Jacques Rousseau, Les Rêveries du promeneur solitaire [Paris: Librairie Générale Française, 1983], 73).

9. Voir Raymond Trousson, Stendhal et Rousseau : Continuité et ruptures (Köln: DME, 1986).

10. Son hédonisme est souligné par une sensibilité envers la peine et le plaisir: "Je ne sais pas ce que je suis: bon, méchant, spirituel, sot. Ce que je sais parfaitement, ce sont les choses qui me font peine ou plaisir, que je désire ou que je hais" (280). En fait, ce sont les plaisirs dans la vie qui rendent l'existence humaine tolérable et parfois même inoubliable: "Pour un tel moment il vaut la peine d'avoir vécu" (413).

11. Effectivement, les freudiens n'ont pas manqué l'occasion de commenter ce passage. Par exemple, Jean Bellemin-Noël observe: "Henry Brulard est précisément cet homme qui proclame EEdipe réincarné; j’ai désiré ma mère à la folie et j'ai souhaité la mort de mon père" (Bellemin-Noel, 59). Voir, aussi, Grazia Merler, "Stendhal dans la perspective adlérienne," Nineteenth-Century French Studies 23 1-2 (1994-5): 108-26, pour une étude psychanalytique très intéressante qui cherche à élucider d'un point de vue adlérien la relation complexe entre l'œuvre de fiction, l'œuvre critique et l'œuvre biographique de Stendhal.

12. Le narrateur nous avait déjà fait savoir que pour lui: "L'amour a toujours été [...] la plus grande des affaires, ou plutôt la seule" (244).

13. Je voudrais exprimer ma reconnaissance à Victor Brombert, pour m'avoir fait découvrir la pensée de Stendhal. 


\section{Works Cited}

Amoss, Benjamin McRae. Time and narrative in Stendhal. Athens: University of Georgia Press, 1992.

Bellemin-Noël, Jean. Biographies du désir: Stendhal-Breton-Leiris. Paris: Presses Universitaires de France, 1988.

Coe, Richard N. "Stendhal, Rousseau, and the Search for the Self." Australian Journal of French Studies 16 (1979): 27-47.

Crouzet, Michel. "Écriture et autobiographie dans la Vie de Henry Brulard."

Stendhal et les problèmes de l'autobiographie. Actes du colloque interuniversitaire (avril 1974). Ed. Victor Del Litto. Grenoble: Presses Universitaires Grenoble, 1976. 105-32.

Del Litto, Victor. Préface. Vie de Henry Brulard par Stendhal. Ed. Del Litto. Genève-Paris: Slatkine Reprints, 1986. i-xii.

Diderot, Denis. Jacques le fataliste. Paris: Librairie Générale Française, 1983.

Didier, Béatrice. Stendhal autobiographe. Paris: Presses Universitaires de France, 1983.

Lejeune, Philippe. "Stendhal et les problèmes de l'autobiographie." Stendhal et les problèmes de l'autobiographie. Actes du colloque interuniversitaire (avril 1974). Ed. Victor Del Litto. Grenoble: Presses Universitaires Grenoble, 1976.21-33.

Merler, Grazia. "Stendhal dans la perspective adlérien." Nineteenth-Century French Studies 231-2 (1994-5): 108-26.

Rousseau, Jean-Jacques. Les Rêveries du promeneur solitaire. Paris: Librairie Générale Française, 1983.

Sartre, Jean-Paul. La Transcendance de l'ego. Paris: Librairie Philosophiques, 1992.

Stendhal.. Vie de Henry Brulard. Paris: Gallimard, 1973.

Talbot, Emile J. Stendhal Revisited. New York: Twayne Publishers, 1993. Trousson, Raymond. Stendhal et Rousseau : Continuité et ruptures. Köln: DME, 1986. 